\title{
Drag and Lift Forces between a Rotating Conductive Sphere and a Cylindrical Magnet
}

\author{
Mark A. Nurge* and Robert C. Youngquist ${ }^{\dagger}$ \\ National Aeronautics and Space Administration, \\ Mail Code: UB-R3, Kennedy Space Center, Florida 32899
}

(Dated: August 17, 2017)

\begin{abstract}
Modeling the interaction between a non-uniform magnetic field and a rotating conductive object allows study of the drag force which is used in applications such as eddy current braking and linear induction motors as well as the transition to a repulsive force that is the basis for magnetic levitation systems. Here, we study the interaction between a non-uniform field generated by a cylindrical magnet and a rotating conductive sphere. Each eddy current in the sphere generates a magnetic field which in turn generates another eddy current, eventually feeding back on itself. A two step mathematics process is developed to find a closed form solution in terms of only two eddy currents. However, the complete solution requires decomposition of the magnetic field into a summation of spherical harmonics, making it more suitable for a graduate level electromagnetism lecture or lab. Finally, the forces associated with these currents are calculated and then verified experimentally.
\end{abstract}




\section{INTRODUCTION}

If a magnet is moved slowly along the surface of a conducting sheet, eddy currents are generated in the sheet that create drag forces, opposite the direction of motion, between the magnet and the currents. These forces are the basis for eddy current braking, ${ }^{1}$ where moving magnets are slowed down as they pass by a conductor, and for linear induction motors, ${ }^{2}$ where a moving magnetic field can strongly propel a conducting sheet (see for example the electromagnetic aircraft launch system, EMALS). ${ }^{3}$ Second order eddy currents are also induced that create an image magnet whose orientation opposes the real magnet. This repulsive force is insignificant at low speeds, but becomes dominant as the magnet is moved rapidly across the sheet, pushing the magnet away from the conducting sheet, i.e., providing increasing lift as the drag force diminishes. The most well-known examples of this are certain embodiments of magnetic railways, i.e., MagLev, ${ }^{4}$ where a moving car floats on a conducting railway.

This transition from drag to lift has been studied ${ }^{5-7}$ but the derivations are typically applied to infinite conducting sheets where an eddy current wake is created. These derivations are pertinent for many real-world applications, but they are concise, often only approximate, and complicated. In the discussion below, a step-by-step derivation will be provided showing how to calculate the drag and repulsion forces between a cylindrical magnet and a rotating solid sphere. We chose this geometry because it allows closed form solutions for all of the electro-magnetic entities involved and can yield insight into the physics behind this force evolution. Some of the details in this derivation are adapted from Heinrich Hertz's doctoral thesis $^{8}$ where he develops a methodology for finding the eddy currents in a rotating sphere when exposed to a constant magnetic field. However, Hertz math is customized, and consequently difficult to follow, since the modern Heaviside notation had not yet occurred. So, from a historical perspective the development given below provides insight into an approach first developed by Hertz in the 1880s when he was only 23.

The derivation provided in this paper is appropriate for a graduate level electromagnetism lecture or lab. The problem requires knowledge of many of the key concepts of electromagnetism and applies them with the goal of modeling and understanding important, but often neglected, topics such as eddy current generation and the interaction between a magnet and a conductor moving at some relative velocity. Our prior papers described the interaction of 
a magnet with a slowly rotating shell ${ }^{9}$, where torques were generated from the drag force but since only the first eddy currents were modeled, lift did not occur; and the interaction of a uniform magnetic field with a rapidly rotating solid sphere ${ }^{10}$, where the symmetry of the uniform magnetic field prevents a lift force from occurring. This paper, describing the interaction of a non-uniform magnetic field, such as that generated by a cylindrical magnet or current loop, with a solid sphere rotating at any speed, does yield lift forces. Also, this is the simplest geometric form where a complete representation of the eddy currents can be obtained and where the results can be expressed in closed form. It should be noted that this complete representation requires the applied magnetic field be decomposed into a summation of spherical harmonic components. This two-dimensional orthogonal series representation may be beyond an undergraduate level, but offers an introduction into complete infinite bases, i.e., Hilbert Spaces, for the graduate student outside of those presented in quantum mechanics. In addition, this paper presents an experiment that can be performed to provide verification of the theoretical predictions.

\section{STARTING CONDITIONS}

A sketch of the problem is shown in Figure 1 with both Cartesian and spherical coordinates that have their origin on the sphere center and are fixed to the laboratory frame of reference. The sphere rotates with a constant angular velocity given by $\vec{\omega}=\omega \hat{z}$ and has a radius given by $R$. It has uniform conductivity, $\sigma$, but has no net charge and is not magnetic, i.e., the relative permeability of the sphere is equal to one. Also, since the sphere is round, the conductivity, as seen from the lab frame, is not time varying (this is important since most rotating objects would cause the conductivity at some location in space to alternate as material entered and then left it). The applied magnetic field, $\vec{B}$, is not time varying and is cylindrically symmetric since we are assuming a round magnet or current loop as the source of the field. The coordinate system has been rotated so that the magnetic field points in the $x$-direction and is symmetric about this axis. Since the magnetic field, angular velocity, and conductivity are not time varying, all deduced parameters such as currents, potential, and electric fields are also not time varying.

We are only concerned with the magnetic field in the volume of the spinning sphere and assume that this applied field has been created by currents (or magnets) outside of the sphere. 


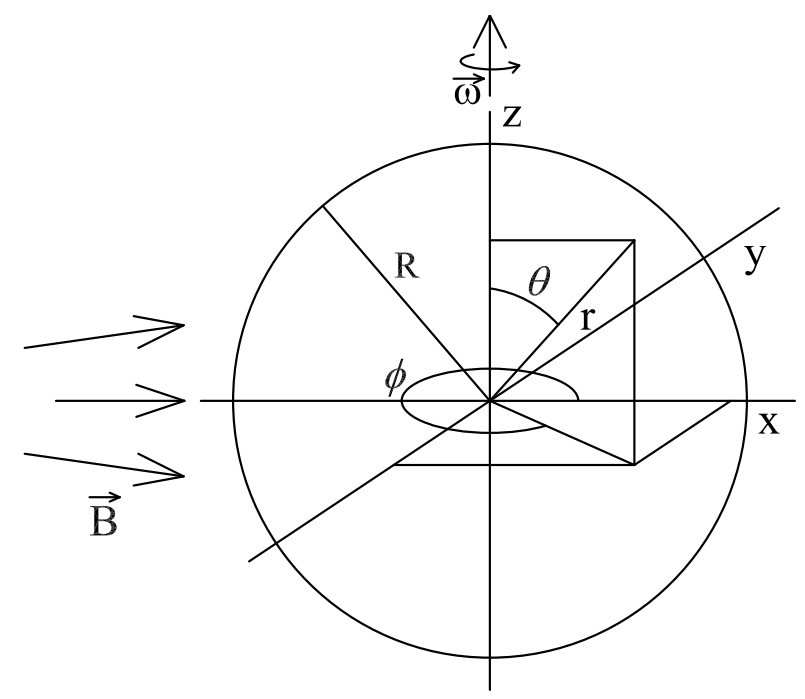

FIG. 1. A magnetic field applied to a rotating solid sphere showing both the Cartesian and spherical coordinate systems used.

Combining this assumption with the time independence of any electric fields causes Ampere's Law to reduce to $\nabla \times \vec{B}=0$. This implies that the magnetic field can be written as the gradient of a magnetic scalar potential, ${ }^{13} \Phi_{M}$, i.e., $\vec{B}=-\nabla \Phi_{M}$. This potential is commonly used in magnetostatics because when combined with Maxwell's equation, $\nabla \cdot \vec{B}=0$, it solves Laplace's Equation, i.e., $\nabla^{2} \Phi_{M}=0$, a well-studied equation. In spherical coordinates this means the magnetic scalar potential can be written as a summation of two orthogonal sets of functions, Legendre functions and trigonometric functions, ${ }^{11}$

$$
\Phi_{M}(r, \theta, \phi)=\sum_{n=0}^{\infty} \sum_{m=0}^{n} b_{n, m} \frac{r^{n}}{R^{n-1}} P_{n}^{m}(\cos \theta) \cos (m \phi) .
$$

This is not the most general form of the solution; polynomial terms that are unbounded at the origin have been dropped and, since the magnetic field is symmetric about the $x$-axis, only cosine terms are retained. Equation 1 is sometimes expressed in terms of Spherical Harmonics, but we chose to use the more explicit form shown. The factors in Eq. 1 have been chosen to simplify the analysis and to give the $b_{n, m}$ coefficients the same units as the magnetic field, i.e., Tesla. Using this decomposition, the magnetic field can now be found allowing an analysis of the problem to begin. 


\section{THE EDDY CURRENTS}

It should be noted that while discussing eddy currents, we are really talking about current densities, but for simplicity will continue to refer to them as currents for the remainder of the paper. In this section, the primary task is to find the currents in the rotating sphere when exposed to an applied magnetic field. Start by using $\vec{B}=-\nabla \Phi$, and the expression in Eq. 1, to find a representation for the magnetic field

$$
\vec{B}=\sum_{n=0}^{\infty} \sum_{m=0}^{n} \vec{B}_{n, m}
$$

where,

$$
\vec{B}_{n, m}=\left(\begin{array}{c}
-b_{n, m}\left(\frac{r}{R}\right)^{n-1} n P_{n}^{m}(\cos \theta) \cos (m \phi) \hat{r} \\
+b_{n, m}\left(\frac{r}{R}\right)^{n-1}\left((1+n) \cos \theta P_{n}^{m}(\cos \theta)+(m-1-n) P_{n+1}^{m}(\cos \theta)\right) \csc \theta \cos (m \phi) \hat{\theta} \\
+b_{n, m} m\left(\frac{r}{R}\right)^{n-1} P_{n}^{m}(\cos \theta) \csc \theta \sin (m \phi) \hat{\phi}
\end{array}\right)
$$

and $\vec{B}_{n, m}$ is the $n, m$ component of the magnetic field. A typical magnetic field of this form is shown in Fig. 2. Recall from Ohm's Law that the current is related to the applied field

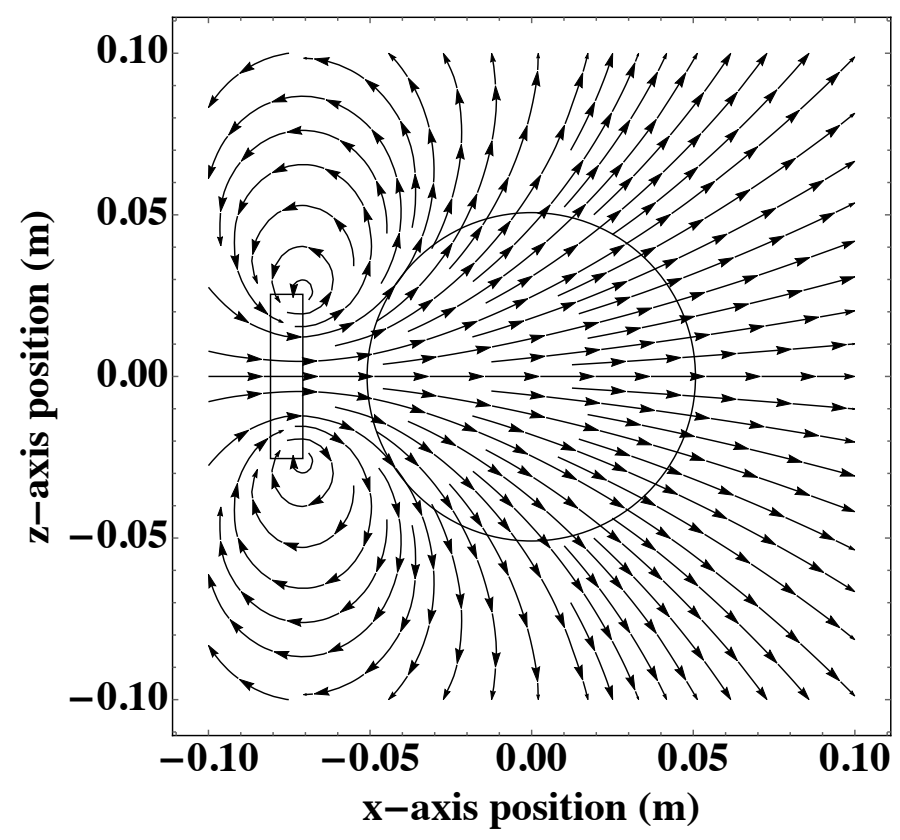

FIG. 2. A representative magnetic field created by a coil, aligned with the $x$-axis near the rotating sphere. 
by

$$
\vec{J}_{n, m}=\sigma\left(\vec{E}_{n, m}+\vec{v} \times \vec{B}_{n, m}\right) .
$$

Using the angular velocity, $\vec{\omega}=\omega \hat{z}$, the velocity of any point in the sphere is given by $\vec{v}=\vec{\omega} \times \vec{r}$. To find $\vec{v} \times \vec{B}_{n, m}$ the magnetic field is converted to Cartesian form, the cross product taken, and the result converted back to spherical coordinates yielding

$$
\begin{aligned}
\vec{v} \times \vec{B}_{n, m}= & -b_{n, m} \frac{\omega r^{n}}{R^{n-1}}\left((1+n) \cos \theta P_{n}^{m}(\cos \theta)+(m-1-n) P_{n+1}^{m}(\cos \theta)\right) \cos (m \phi) \hat{r} \\
& -b_{n, m} \frac{\omega r^{n}}{R^{n-1}} n \sin \theta P_{n}^{m}(\cos \theta) \cos (m \phi) \hat{\theta}
\end{aligned}
$$

Now, recall that conservation of charge states that the divergence of the current is equal to the change in the charge density with time, i.e., $\nabla \cdot \vec{J}=\partial \rho / \partial t$. However, since all of the parameters in this problem are time independent this becomes $\nabla \cdot \vec{J}=0$. So, taking the divergence of Eq. 3 and using Eq. 4 yields

$$
\begin{aligned}
\nabla \cdot \vec{E}_{n, m} & =-\nabla \cdot\left(\vec{v} \times \vec{B}_{n, m}\right) \\
& =2 b_{n, m} \frac{\omega r^{n-1}}{R^{n-1}}\left((1+2 n) \cos \theta P_{n}^{m}(\cos \theta)+(m-1-n) P_{n+1}^{m}(\cos \theta)\right) \cos (m \phi)
\end{aligned}
$$

The electric potential, $V$, is related to the electric field by $\vec{E}=-\nabla V$ so Eq. 5 becomes a Poisson Equation

$$
\nabla^{2} V_{n, m}=\nabla \cdot\left(\vec{v} \times \vec{B}_{n, m}\right) .
$$

If we can find the electric potential, then we can find the electric field and then the induced currents in the sphere created by the applied field. To do this we need boundary conditions on $V$ and these are that the potential must be bounded throughout space, it must be continuous across the sphere outer boundary, and no currents can leave the sphere. This current boundary condition is physically reasonable - current cannot flow out of the sphereand it is commonly used to solve eddy current problems. However, it is not immediately obvious that it is pertinent since the current is not explicit in Eq. 6. But, if we insist that the radial component of the current at the sphere boundary, $J_{r}(R)=0$, then Eq. 3 yields

$$
\sigma\left(E_{r}(R)+(\vec{v} \times \vec{B})_{r}(R)\right)=0 \Rightarrow \frac{\partial V}{\partial r}(R)=(\vec{v} \times \vec{B})_{r}(R) .
$$

This results in a Neumann boundary condition on the potential (we have dropped the $m, n$ subscript to show the vector component index). Integrating the radial portion of Eq. 4, the electric potential inside of the sphere becomes

$$
V_{m, n}=-b_{n, m} \frac{\omega r^{n+1}}{(n+1) R^{n-1}}\left((1+n) \cos \theta P_{n}^{m}(\cos \theta)+(m-1-n) P_{n+1}^{m}(\cos \theta)\right) \cos (m \phi)
$$


and the homogenous solution of Eq. 6 is used outside of the sphere to ensure continuity of the potential across the sphere boundary. Using this form for the potential, the electric field can be found and, from Eq. 3, the current can be obtained;

$$
\begin{aligned}
\vec{J}_{n, m}= & -\sigma \omega b_{n, m} \frac{r^{n}}{R^{n-1}} \frac{m}{(n+1)} \csc \theta\left(m P_{n}^{m}(\cos \theta) \cos (m \phi) \hat{\theta}\right. \\
& \left.+\left((1+n) \cos \theta P_{n}^{m}(\cos \theta)+(m-1-n) P_{n+1}^{m}(\cos \theta)\right) \sin (m \phi) \hat{\phi}\right)
\end{aligned}
$$

As first pointed out by Hertz, there are no radial current components; all of the current flows along shells, forming closed loops since the divergence is zero. Figure 3 shows a representative form for this current, looking along the $x$-axis. The velocity of the spheres surface shown in this Figure is in the positive $y$-direction and the magnetic field is into the paper yielding an upward force that results in the current shown.

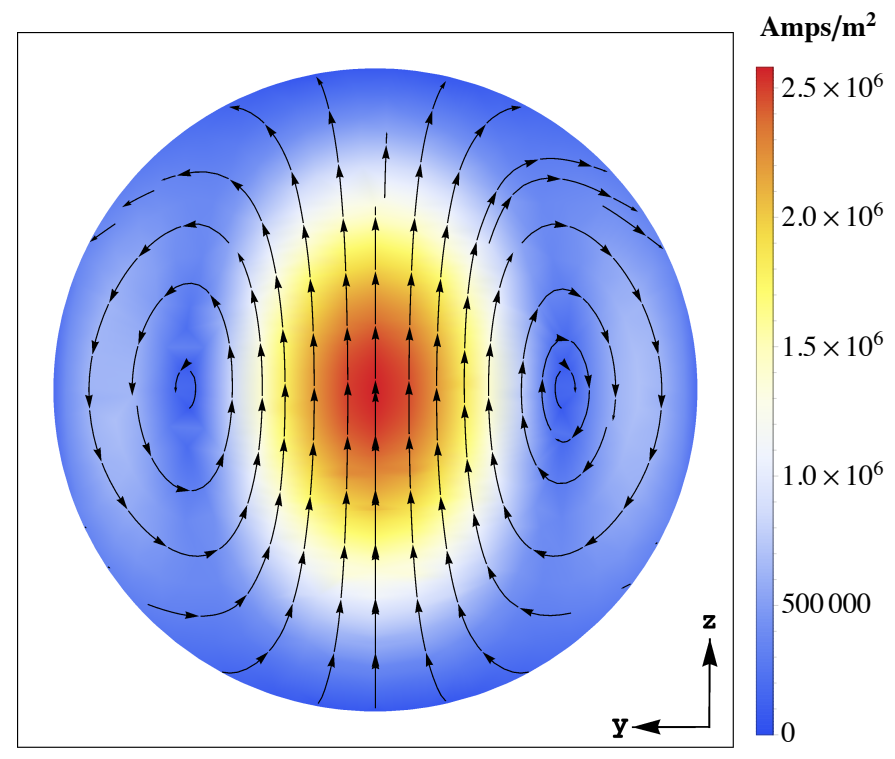

FIG. 3. A typical form for the eddy current generated by the rotating sphere in the presence of a magnetic field aligned with the $x$-axis. This view is looking from the magnet in the positive $x$-direction with the origin at the sphere center.

The eddy current shown in Eq. 9 is generated by the applied magnetic field interacting with the rotating conducting sphere, but it is not the total current. This eddy current generates a magnetic field within the sphere that, through the same process shown above, yields a second eddy current. Then the second eddy current generates another magnetic 
field which yields a third eddy current, and, as will be shown below, this third eddy current is of the same form as the first eddy current but of opposite sign. So the third eddy current acts to cancel the eddy current in Eq. 9, yielding a negative feedback loop.

To obtain the total current within the sphere, we will take the form of the current shown in Eq. 9 and generalize it by making the radial dependence an unknown function and the goal of the analysis will be to find this function. Once it is obtained, then the second eddy current can be found and the forces and torques on the sphere can be calculated. This insight, that the current shown in Eq. 9 can be generalized in this fashion-only changing the radial dependence - is due to the 23-year-old Hertz. ${ }^{8}$

Let's start with a general form of the first eddy current, $\vec{J}_{n, m}^{1}$, which is equal to the current caused by the applied field, $\vec{J}_{n, m}$, defined by Eq. 9 , added to the third eddy current, $\vec{J}_{n, m}^{3}$,

$$
\begin{aligned}
\vec{J}_{n, m}^{1}= & \vec{J}_{n, m}+\vec{J}_{n, m}^{3} \\
= & -\sigma \omega b_{n, m} c_{n, m}(r) r \csc \theta\left(m P_{n}^{m}(\cos \theta) \cos (m \phi) \hat{\theta}\right. \\
& \left.+\left((1+n) \cos \theta P_{n}^{m}(\cos \theta)+(m-1-n) P_{n+1}^{m}(\cos \theta)\right) \sin (m \phi) \hat{\phi}\right) .
\end{aligned}
$$

$c_{n, m}(r)$ is a dimensionless function of the radial distance and the goal of the analysis is to find this function. Note that $c_{n, m}(r)$ and the other current related scalar functions derived below are functions of the subscripts $n$ and $m$. If there is no third eddy current, then $c_{n, m}(r)=(m /(n+1))(r / R)^{n-1}$, which corresponds to the limiting solution when the sphere is small or rotates slowly and must emerge from the more general solution. The process will be to find the magnetic field generated by the current $\vec{J}_{n, m}^{1}$ and then find the second order eddy current, $\vec{J}_{n, m}^{2}$, generated by that field. We then do this again to find the third order eddy current, which will allow the feedback loop to be closed and the function $c_{n, m}(r)$ to be found.

In order to find the magnetic field $\vec{B}_{n, m}^{2}$ generated by the current, $\vec{J}_{n, m}^{1}$, use the vector potential equation, $\nabla^{2} \vec{A}_{n, m}^{2}=-\mu_{0} \vec{J}_{n, m}^{1}$, (where $\mu_{0}$ is the magnetic constant, $4 \pi \times 10^{-7} \mathrm{~A} / \mathrm{m}^{2}$ ) to find $\vec{A}_{n, m}^{2}$ and then use $\vec{B}_{n, m}^{2}=\nabla \times \vec{A}_{n, m}^{2}$ to find the field. Note that we use superscript " 2 " here to indicate that these are the second set of magnetic fields and potentials and not that these entities are squared. To solve the vector potential equation, the current and the vector potential are first both put into the form $\vec{J}(r, \theta, \phi)=J_{x}(r, \theta, \phi) \hat{x}+J_{y}(r, \theta, \phi) \hat{y}+J_{z}(r, \theta, \phi) \hat{z}$ so that the Laplacian operator can be applied to each component of the vector functions, but the dependence is still in spherical coordinates so that the boundary conditions can be 
easily handled. Doing this to the current yields

$$
\begin{aligned}
& \vec{J}_{n, m}^{1}=\sigma \omega b_{n, m} c_{n, m}(r) r\left(\frac{1}{2}(\right.\left(n+n^{2}+m-m^{2}\right) P_{n}^{m-1}(\cos \theta) \cos ((m-1) \phi) \\
&\left.+P_{n}^{m+1}(\cos \theta) \cos ((m+1) \phi)\right) \hat{x} \\
&+\frac{1}{2}\left(-\left(n+n^{2}+m-m^{2}\right) P_{n}^{m-1}(\cos \theta) \sin ((m-1) \phi)+P_{n}^{m+1}(\cos \theta) \sin ((m+1) \phi)\right) \hat{y} \\
&\left.+m P_{n}^{m}(\cos \theta) \cos (m \phi) \hat{z}\right) .
\end{aligned}
$$

This expression shows that in a Cartesian decomposition the current components are all in the forms of spherical harmonics (note the order of the trigonometric functions matches the superscript of the associated Legendre functions). This is important because the Laplacian of a spherical harmonic returns a modified radial function times the same spherical harmonic. So, the inhomogeneous portion of the vector scalar potential can be put into the same form as the current but with a different radial dependence, i.e.,

$$
\begin{aligned}
& \vec{A}_{n, m}^{2}= b_{n, m} g_{n, m}(r) r\left(\frac { 1 } { 2 } \left(\left(n+n^{2}+m-m^{2}\right) P_{n}^{m-1}(\cos \theta) \cos ((m-1) \phi)\right.\right. \\
&\left.+P_{n}^{m+1}(\cos \theta) \cos ((m+1) \phi)\right) \hat{x} \\
&+\frac{1}{2}\left(-\left(n+n^{2}+m-m^{2}\right) P_{n}^{m-1}(\cos \theta) \sin ((m-1) \phi)+P_{n}^{m+1}(\cos \theta) \sin ((m+1) \phi)\right) \hat{y} \\
&\left.+m P_{n}^{m}(\cos \theta) \cos (m \phi) \hat{z}\right)
\end{aligned}
$$

where the new dimensionless radial function, $g_{n, m}(r)$, is given by

$$
r^{2} g_{n, m}^{\prime \prime}(r)+4 r g_{n, m}^{\prime}(r)+\left(2-n-n^{2}\right) g_{n, m}(r)+r^{2} \mu_{0} \sigma \omega c(r)=0 .
$$

However, the form for the vector potential shown in Eq. 12 is not complete. The homogeneous solutions must be added in order to obtain continuity of each component of the vector potential, $\vec{A}_{n, m}^{2}$, and each component of the magnetic field, $\vec{B}_{n, m}^{2}$, across the sphere boundary where $r=R$. Since each spherical harmonic in Eq. 12 yields a corresponding homogeneous spherical harmonic both inside and outside of the sphere, there are ten coefficients to be found and determining them is a tedious process. In addition, Eq. 13 for $g_{n, m}(r)$ has two homogenous solutions, one of which is determined by stating that $g_{n, m}(r)$ must be bounded at the origin. The other, when placed into the complete solution for $\vec{A}_{n, m}^{2}$ drops out, having no effect on $\vec{A}_{n, m}^{2}$, so we are free to choose an arbitrary form for this second homogeneous solution which simplifies the resulting form for both $\vec{A}_{n, m}^{2}$ and for $g_{n, m}(r)$. The final result 
for the vector potential in spherical coordinates is

$$
\vec{A}_{n, m}=\left\{\begin{array}{l}
-b_{n, m} g_{n, m}(r) r m \csc \theta P_{n}^{m}(\cos \theta) \cos (m \phi) \hat{\theta} \\
+b_{n, m} g_{n, m}(r) r\left(m \cot \theta P_{n}^{m}(\cos \theta)+P_{n}^{m+1}(\cos \theta)\right) \sin (m \phi) \hat{\phi}, \quad r<R \\
-b_{n, m} g_{n, m}(R) \frac{R^{n+2}}{r^{n+1}} m \csc \theta P_{n}^{m}(\cos \theta) \cos (m \phi) \hat{\theta} \\
+b_{n, m} g_{n, m}(R) \frac{R^{n+2}}{r^{n+1}}\left(m \cot \theta P_{n}^{m}(\cos \theta)+P_{n}^{m+1}(\cos \theta)\right) \sin (m \phi) \hat{\phi}, \quad r>R
\end{array}\right.
$$

and for $g_{n, m}(r)$ is

$$
g_{n, m}(r)=\mu_{0} \sigma \omega\left(r^{n-1} \int_{r}^{R} \frac{c_{n, m}(t)}{(1+2 n) t^{n-2}} d t+\frac{1}{r^{n+2}} \int_{0}^{r} \frac{c_{n, m}(t) t^{n+3}}{(1+2 n)} d t\right) .
$$

We have converted the vector potential back to a spherical basis so that curl can be taken to yield the second magnetic field, $\vec{B}_{n, m}^{2}$. For the volume within the sphere this is given by

$$
\begin{aligned}
& \vec{B}_{n, m}^{2}=-b_{n, m} n(1+n) g_{n, m}(r) P_{n}^{m}(\cos \theta) \sin (m \phi) \hat{r} \\
& +b_{n, m}\left(2 g_{n, m}(r)+r g_{n, m}^{\prime}(r)\right) \csc \theta\left((1+n) \cos \theta P_{n}^{m}(\cos \theta)+(m-1-n) P_{n+1}^{m}(\cos \theta)\right) \sin (m \phi) \hat{\theta} \\
& \quad-b_{n, m} m\left(2 g_{n, m}(r)+r g_{n, m}^{\prime}(r)\right) \csc \theta P_{n}^{m}(\cos \theta) \cos (m \phi) \hat{\phi} \quad(16)
\end{aligned}
$$

Comparing this to the applied field, the radial dependence is of course different, but the angular dependence is identical except that $\cos (m \phi)$ has become $-\sin (m \phi)$ and $\sin (m \phi)$ has become $\cos (m \phi)$ indicating a net rotation of the field about the $z$-axis.

Following the same procedure shown above for the applied magnetic field, we next need to find $\vec{v} \times \vec{B}_{n, m}^{2}$. So, convert the magnetic field in Eq. 16 to Cartesian form, take the cross product, and convert the result back to spherical coordinates yielding

$$
\begin{aligned}
& \vec{v} \times \vec{B}_{n, m}^{2}=-b_{n, m} \omega r\left(2 g_{n, m}(r)+r g_{n, m}{ }^{\prime}(r)\right)\left((n+1) \cos \theta P_{n}^{m}(\cos \theta)\right. \\
&\left.+(m-1-n) P_{n+1}^{m}(\cos \theta)\right) \sin (m \phi) \hat{r} \\
& \quad-b_{n, m} \omega r g_{n, m}(r) n(1+n) \sin \theta P_{n}^{m}(\cos \theta) \sin (m \phi) \hat{\theta} .
\end{aligned}
$$

The potential inside of the sphere is obtained by integrating the radial portion of Eq. 17, using the current boundary condition, yielding

$$
V_{n, m}^{2}=-b_{n, m} \omega r^{2} g_{n, m}(r)\left((1+n) \cos \theta P_{n}^{m}(\cos \theta)+(m-1-n) P_{n+1}^{m}(\cos \theta)\right) \sin (m \phi),
$$


which is very similar to the potential generated by the applied field shown in Eq 8 .

The electric field can be found from the potential and, combining this with the cross product result from Eq. 17, the second eddy current (see Ohm's Law in Eq. 3) is obtained:

$$
\begin{aligned}
& \vec{J}_{n, m}^{2}=-\sigma \omega m^{2} b_{n, m} r g_{n, m}(r) \csc \theta P_{n}^{m}(\cos \theta) \sin (m \phi) \hat{\theta} \\
& +\sigma \omega m b_{n, m} r g_{n, m}(r) \csc \theta\left((1+n) \cos \theta P_{n}^{m}(\cos \theta)+(m-1-n) P_{n+1}^{m}(\cos \theta)\right) \cos (m \phi) \hat{\phi} .
\end{aligned}
$$

The impressive result here is that the second eddy current is nearly identical to the first current shown in Eq. 10. The function $c_{n, m}(r)$ has become $m g_{n, m}(r)$ and the $\phi$ dependence has shifted; $\cos (m \phi)$ has become $\sin (m \phi)$ and $\sin (m \phi)$ has become $-\cos (m \phi)$, indicating a rotation of the vector field about the $z$-axis. Figure 4 shows a representative second eddy current. The circular shape taken by this second eddy current causes it to generate a magnetic field that opposes the applied magnetic field.

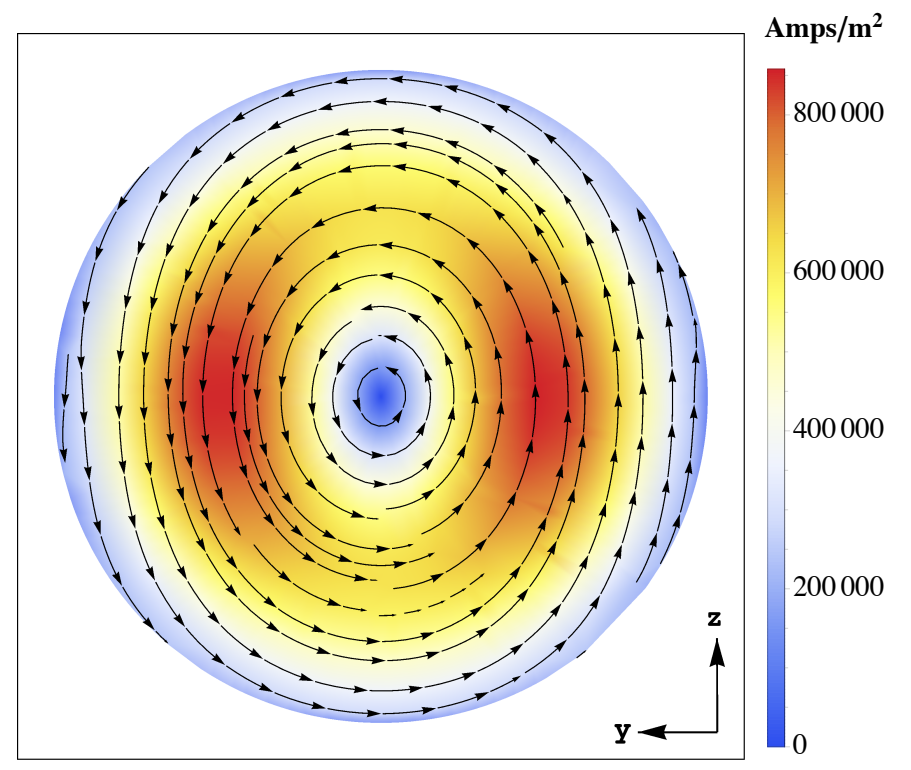

FIG. 4. A typical form for the second eddy current generated by the rotating sphere in the presence of a magnetic field aligned with the $x$-axis. This view is looking from the magnet in the positive $x$-direction with the origin at the sphere center.

In order to find the third eddy current, $\vec{J}_{n, m}^{3}$, start with the current shown in Eq. 19 and repeat the process from Eq. 10 through 19. The $m g_{n, m}(r)$ will become $m^{2} k_{n, m}(r)$, the 
$\cos (m \phi)$ will become $\sin (m \phi)$, and the $\sin (m \phi)$ will become $-\cos (m \phi)$, yielding

$$
\begin{aligned}
& \vec{J}_{n, m}^{3}=\sigma \omega b_{n, m} m^{3} k_{n, m}(r) r \csc \theta P_{n}^{m}(\cos \theta) \cos (m \phi) \hat{\theta} \\
& +\sigma \omega b_{n, m} m^{2} k_{n, m}(r) r \csc \theta\left((1+n) \cos \theta P_{n}^{m}(\cos \theta)+(m-1-n) P_{n+1}^{m}(\cos \theta)\right) \sin (m \phi) \hat{\phi}
\end{aligned}
$$

and

$$
k_{n, m}(r)=\mu_{0} \sigma \omega\left(r^{n-1} \int_{r}^{R} \frac{g_{n, m}(t)}{(1+2 n) t^{n-2}} d t+\frac{1}{r^{n+2}} \int_{0}^{r} \frac{g_{n, m}(t) t^{n+3}}{(1+2 n)} d t\right) .
$$

This third eddy current has nearly the same form as the first eddy current (Eq. 10); the only significant difference being a sign change, indicating negative feedback, which is needed to achieve a stable solution.

Recall that the starting current is the sum of the current created by the applied field and the third eddy current, i.e., $\vec{J}_{n, m}^{1}=\vec{J}_{n, m}+\vec{J}_{n, m}^{3}$. So, combining Eqs. 9, 10, and 20 we obtain

$$
c_{n, m}(r)=(m /(n+1))(r / R)^{n-1}-m^{2} k_{n, m}(r) .
$$

The functions $c_{n, m}(r), g_{n, m}(r)$, and $k_{n, m}(r)$ are also related by the second order differential equations (see Eq. 13)

$$
\begin{aligned}
& r^{2} g_{n, m}{ }^{\prime \prime}(r)+4 r g_{n, m}{ }^{\prime}(r)+\left(2-n-n^{2}\right) g_{n, m}(r)+r^{2} \mu_{0} \sigma \omega c(r)=0 \\
& r^{2} k_{n, m}^{\prime \prime}(r)+4 r k_{n, m}^{\prime}(r)+\left(2-n-n^{2}\right) k_{n, m}(r)+r^{2} \mu_{0} \sigma \omega g(r)=0 .
\end{aligned}
$$

Solving Eq 22 for the function $k_{n, m}(r)$ and substituting this into the second equation in Eq. 23 yields

$$
r^{2} c_{n, m}^{\prime \prime}(r)+4 r c_{n, m}^{\prime}(r)+\left(2-n-n^{2}\right) c_{n, m}(r)-r^{2} \mu_{0} \sigma \omega m^{2} g_{n, m}(r)=0
$$

Now Eq. 24 can be solved for $g_{n, m}(r)$ and substituted into the first expression in Eq. 23 to yield a fourth order differential equation in $c_{n, m}(r)$

$$
\begin{aligned}
r^{4} c_{n, m}{ }^{(4)}(r)+8 r^{3} c_{n, m}{ }^{(3)}(r)-2\left(-6+n+n^{2}\right) r^{2} c_{n, m}{ }^{\prime \prime}(r)-4 n(1+n) r c_{n, m}{ }^{\prime}(r) & \\
& +\left(-2 n-n^{2}+2 n^{3}+n^{4}+\alpha_{m}{ }^{4} r^{4}\right) c_{n, m}(r)=0,
\end{aligned}
$$

where $\alpha_{m}^{2}=\mu_{0} \sigma \omega m$ and $\alpha_{m}$ has units of inverse length. This fourth order differential equation has four solutions corresponding to each of the Kelvin functions, but two of these are unbounded at the origin and will be dropped since we require bounded solutions at the 
origin. However, for the case of a thick walled rotating shell with no material at the origin all four solutions would be required to meet the boundary conditions. So, the general solution to Eq. 25 is

$$
c_{n, m}(r)=\left(c_{1, n, m} \operatorname{ber}_{n+1 / 2}\left(\alpha_{m} r\right)+c_{2, n, m} \operatorname{bei}_{n+1 / 2}\left(\alpha_{m} r\right)\right) /\left(\alpha_{m} r\right)^{3 / 2}
$$

Using the second equation in Eq. $24 g(r)$ can be found

$$
g(r)=(1 / m)\left(c_{2, n, m} \operatorname{ber}_{n+1 / 2}\left(\alpha_{m} r\right)-c_{1, n, m} \operatorname{bei}_{n+1 / 2}\left(\alpha_{m} r\right)\right) /\left(\alpha_{m} r\right)^{3 / 2}
$$

Since the general solutions shown in Eq. 26 and Eq. 27 simply yield each other when substituted into the differential equations, the coefficients cannot be found using the differential relationships. Instead, the integral relations, Eq. 15 and Eq. 21, along with Eq. 22, are used. The expressions shown in Eq 26 and Eq 27 are substituted into these integral relations and two linear expressions for the coefficients, $c_{1}$ and $c_{2}$, are obtained. Solving these yields the following expressions for the coefficients,

$$
\begin{aligned}
& c_{1, n, m}=-\frac{(1+2 n) \sqrt{\alpha_{m} R}\left(\operatorname{bei}_{n-1 / 2}\left(\alpha_{m} R\right)+\operatorname{ber}_{n-1 / 2}\left(\alpha_{m} R\right)\right)}{\sqrt{2}(n+1)\left(\operatorname{bei}_{n-1 / 2}^{2}\left(\alpha_{m} R\right)+\operatorname{ber}_{n-1 / 2}^{2}\left(\alpha_{m} R\right)\right)} \\
& c_{2, n, m}=-\frac{(1+2 n) \sqrt{\alpha_{m} R}\left(\operatorname{bei}_{n-1 / 2}\left(\alpha_{m} R\right)-\operatorname{ber}_{n-1 / 2}\left(\alpha_{m} R\right)\right)}{\sqrt{2}(n+1)\left(\operatorname{bei}_{n-1 / 2}^{2}\left(\alpha_{m} R\right)+\operatorname{ber}_{n-1 / 2}^{2}\left(\alpha_{m} R\right)\right)} .
\end{aligned}
$$

Combining Eqs. 26-28 and recalling that $\alpha_{m}=\sqrt{\mu_{0} \sigma \omega m}$ yields the two eddy currents, $\vec{J}_{n, m}^{1}$ and $\vec{J}_{n, m}^{2}$, from Eqs. 10 and 19. The sum of these two current densities over all values of $n$ and $m$ yields the total current density, $\vec{J}_{T}$, in the sphere, where

$$
\begin{aligned}
& \vec{J}^{1}=\sum_{n=0}^{\infty} \sum_{m=0}^{n} \vec{J}_{n, m}^{1} \\
& \vec{J}^{2}=\sum_{n=0}^{\infty} \sum_{m=0}^{n} \vec{J}_{n, m}^{2} \\
& \vec{J}_{T}=\vec{J}^{1}+\vec{J}^{2} .
\end{aligned}
$$

\section{THE NET FORCE}

The primary goal of this paper is to obtain the drag and lift forces between the source coil or magnet and the rotating sphere. However, there are several sources of force distributions within the sphere and only a subset yields a non-zero net force. For example, as shown above, the rotation of the sphere in the presence of the applied magnetic field yields forces that cause currents to flow. However, since there is no net charge on the sphere, for every 
force on an electron there is an equal and opposite force on a proton. So, these forces exert no net force on the sphere.

Another source of force is the interaction between the currents themselves. Every differential current element in the sphere feels a force from its interaction with every other differential current element. However, between any two current elements this force is equal and opposite, i.e., the force one current element generates on a second current element is equal to, but opposite, the force the second current element generates on the first one. So if the current elements are both inside of the sphere their net force on the sphere is zero. This is not to say that these forces are small. The internal stress inside of a current can be equated to those inside of a pressure vessel. But the net force between currents $\vec{J}^{1}$ and $\vec{J}^{2}$, or between themselves, is zero because they are all located within the sphere.

The only remaining source of force is the interaction between the current that generated the applied field and the currents inside of the sphere. As before, this interaction yields equal and opposite forces, but one acts on the sphere and one on the coil or magnet that generated the applied field, so these forces affect separate entities that can respond to that force. In practice, this interaction is usually described through the magnetic field generated by one of the currents and the choice is arbitrary. In the present case, the currents and fields inside of the sphere are known and the sphere has useful symmetry so we choose to use the applied field, $\vec{B}$, inside of the sphere and the currents, $\vec{J}^{1}$ and $\vec{J}^{2}$. So, the total net force on the sphere, with an equal but opposite force on the magnet is given by the Lorentz Force Law:

$$
\vec{F}=\int_{\text {sphere }} \vec{J}_{T} \times \vec{B} d \tau .
$$

The total force can be calculated by finding the summations in Eqs. 2, 10, 19, and 29 and substituting the results into Eq. 30. It is recommended that the reader convert each of the vectors over to a Cartesian basis but maintain spherical coordinates as in Eq. 11 to simplify the computations. With the exception of the radial integral, much of the force calculation can be done symbolically, but it is a long tedious process with little physical insight to be gained. Our recommended approach is to decompose the incident magnetic field to find the coefficients, $b_{n, m}$ (as discussed in the next section) and substitute them into the expressions to find the forces numerically. The first current, $\vec{J}^{1}$, will interact with the incoming field and produce a force component that is only in the $y$-direction. So, if only the first current 
is present then the magnet will feel a force pulling it along in the direction of the spheres velocity, while the sphere will feel an equal but opposite force. The force on the sphere will be concentrated near the magnet and, since it is not directed through the center of mass of

the sphere, will generate a torque that will try to slow down the sphere. ${ }^{9}$ The force due to the second eddy current, $\vec{J}^{2}$, is directed only along the $x$-direction. It acts as a repulsive force, pushing the magnet away from the rotating sphere.

\section{MAGNETIC FIELD DECOMPOSITION}

In order to calculate the forces, the coefficients $b_{n, m}$ must be found. We chose to use the radial portion of the magnetic field expression shown in Eq. 2, rather than the magnetic scalar potential, because the magnetic field is often known and is continuous.

$$
B_{r}=-\sum_{n=0}^{\infty} \sum_{m=0}^{n} b_{n, m}\left(\frac{r}{R}\right)^{n-1} n P_{n}^{m}(\cos \theta) \cos (m \phi)
$$

Start by multiplying both sides of Eq. 31 by $\cos \left(m^{\prime} \phi\right)$ where $m^{\prime}$ is an integer between 0 and $n$ and integrate $\phi$ from 0 to $2 \pi$. This integral is zero unless $m=m^{\prime}$, in which case it is equal to either $2 \pi$ when $m=0$ or $\pi$ when $m \neq 0$.

$$
\int_{0}^{2 \pi} \cos (m \phi) B_{r}(r, \theta, \phi) d \phi=-\nu(m) \sum_{n=0}^{\infty} \pi b_{n, m}\left(\frac{r}{r_{2}}\right)^{n-1} n P_{n}^{m}(\cos \theta),
$$

where $\nu(m)=1$ if $m \neq 0$ and equals 2 if $m=0$. Now multiply both sides by $P_{n^{\prime}}^{m}(\cos \theta) \sin \theta$ where $n^{\prime}$ is a positive integer and integrate $\theta$ from 0 to $\pi$. The integral on the right side is zero unless $n=n^{\prime}$ in which case

$$
\begin{aligned}
\int_{0}^{\pi} \int_{0}^{2 \pi} \cos (m \phi) B_{r}(r, \theta, \phi) P_{n}^{m}(\cos \theta) \sin \theta d \phi d \theta & \\
& =-\nu(m) \pi b_{n, m}\left(\frac{r}{r_{2}}\right)^{n-1} \frac{2 n(n+m) !}{(2 n+1)(n-m) !}
\end{aligned}
$$

Equation 33 is true for any value of $r$ so we choose $r=r_{2}$ and then rearrange to solve for the coefficients, yielding

$$
b_{n, m}=-\frac{(2 n+1)(n-m) !}{\nu(m) 2 \pi n(n+m) !} \int_{0}^{\pi} \int_{0}^{2 \pi} B_{r}(r, \theta, \phi) P_{n}^{m}(\cos \theta) \sin \theta \cos (m \phi) d \phi d \theta .
$$


Note that the $b_{n, m}$ coefficients must be finite so $n \neq 0$. Also, any $b_{n, m}$ that appear in the equations above that have negative $n$ or $m$ values or $m$ great than $n$ are defined to be equal to zero.

\section{THE EXPERIMENT}

In order to test the theory, we positioned a cylindrical rare-earth magnet near a rotating solid sphere and measured the two force components. The sphere was $0.051 \mathrm{~m}$ (2 inches) in radius and composed of aluminum with a conductivity of $2.3 \times 10^{7} \mathrm{~S} / \mathrm{m}$. It was mounted with bearings on both the top and bottom and rotated up to $60 \mathrm{~Hz}$. We strongly encourage eye protection be worn when the sphere is rotating and recommend a protective shield or cage be placed over the sphere and motor. Slight misalignments can cause resonances that may lead to bearing failure.

A black cloth was attached to the sphere, so that a light source and detector could monitor the reflectivity of the sphere and determine its rotational speed. A force gage (Imada DS2-1) was used to measure the lateral, or drag, force and the repulsive, or lift, force (see Figure 2 for the configuration used to measure the repulsive force). The rare earth magnet was $0.025 \mathrm{~m}$ (1 inch) in radius and $0.013 \mathrm{~m}$ ( 0.5 inches) thick and the center of its face was located $0.02 \mathrm{~m}$ (0.79 inches) from the spheres surface. The magnet's field was measured using a Gauss probe and since the magnet is relatively thin, it was modeled as a round current loop ${ }^{18}$ with an equivalent current of 9600 Amps. The magnetic field of a round current loop is given in Eq. A4, but in order to use this equation it had to be converted to Cartesian form, rotated so the loop would be aligned with the $x$-axis, shifted back away from the sphere, and then converted back to spherical coordinates so that the $b_{n, m}$ components in Eq. 34 could be found.

Data was taken and compared with the theoretical model discussed above and the results are provided in Fig. 6 and Fig. 7. The match is remarkably good below $30 \mathrm{~Hz}$ but begins to deviate above this frequency. One explanation for the variation is that both forces are present on the sensor, even though we are only measuring one axis at a time. While measuring the repulsive force, a side load exists that peaks in the 30-60 Hz range causing some flex in the shaft and changing the alignment of the magnet slightly. A similar effect is present in the measurement of the lateral force, where the repulsive force continues to grow and will push 


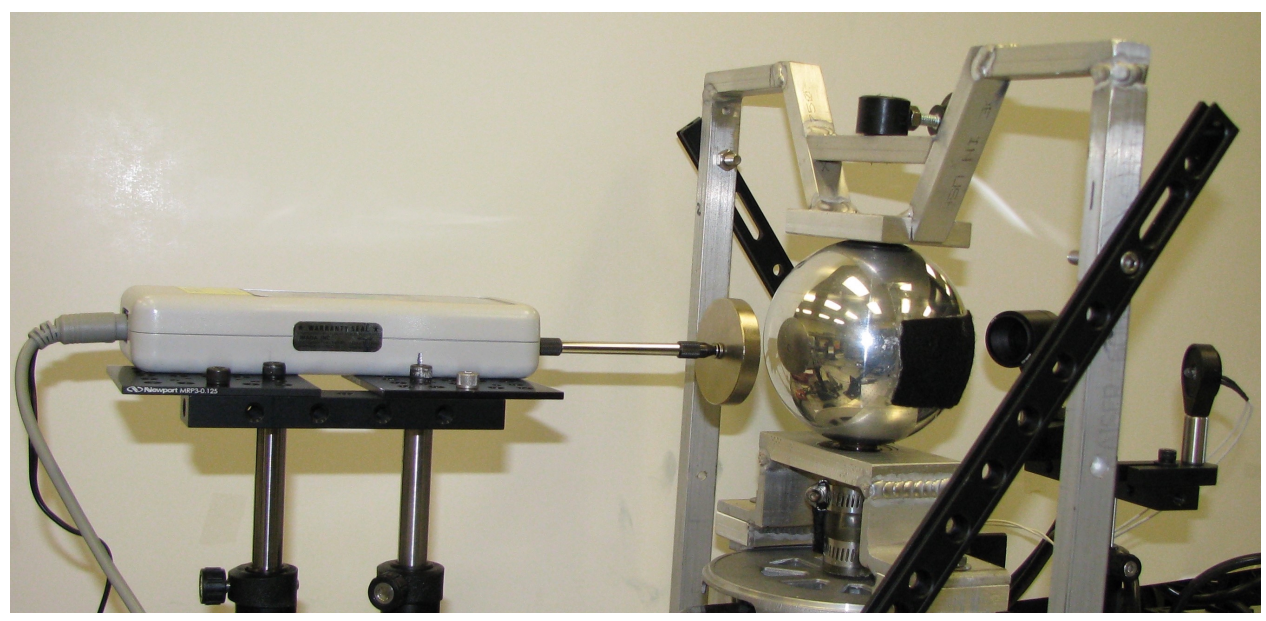

FIG. 5. The experimental configuration used to measure the repulsive force between a spinning solid sphere and a rare earth magnet.

the magnet further away from the sphere, thereby decreasing the lateral force measured by the sensor. Additionally, the sphere experiences a resonance when spinning up through $30-50 \mathrm{~Hz}$ that causes quite a bit of vibration and movement of the sphere. This is most noticeable in the data set for the lateral force measurement.

The models were fit by allowing the distance between the magnet and the sphere to vary. The best fit for the repulsive force model occurred when the magnet was assumed to be $0.017 \mathrm{~m}$ away versus the $0.02 \mathrm{~m}$ as configured. Likewise, the lateral force model gave a best fit for a distance of $0.021 \mathrm{~m}$ versus the $0.02 \mathrm{~m}$ as configured. A straight edged cardboard shim was used to adjust the distance between the sphere and magnet for the test. Its not unreasonable that there may be a few millimeters of error in the alignment, since perfect alignment of the center of the magnet with the outer edge of a curved surface is difficult to achieve. Additionally, the side loads and vibrations also vary the alignment during the test. Another potential source of error is that close up, the neodymium magnets magnetic field will vary some from the idealized coil model we used to represent the magnet.

Overall, the data closely agrees with theory. However, Figures 6 and 7 don't show the full extent of the variations in the forces with increased rotational frequency. In fact, as discussed in our previous paper, the forces are actually dependent on a combination of the sphere's radius, electrical conductivity, magnetic permeability, and the rotational frequency. The theoretical forces for our test sphere are plotted below in Figure 8 as a function of 


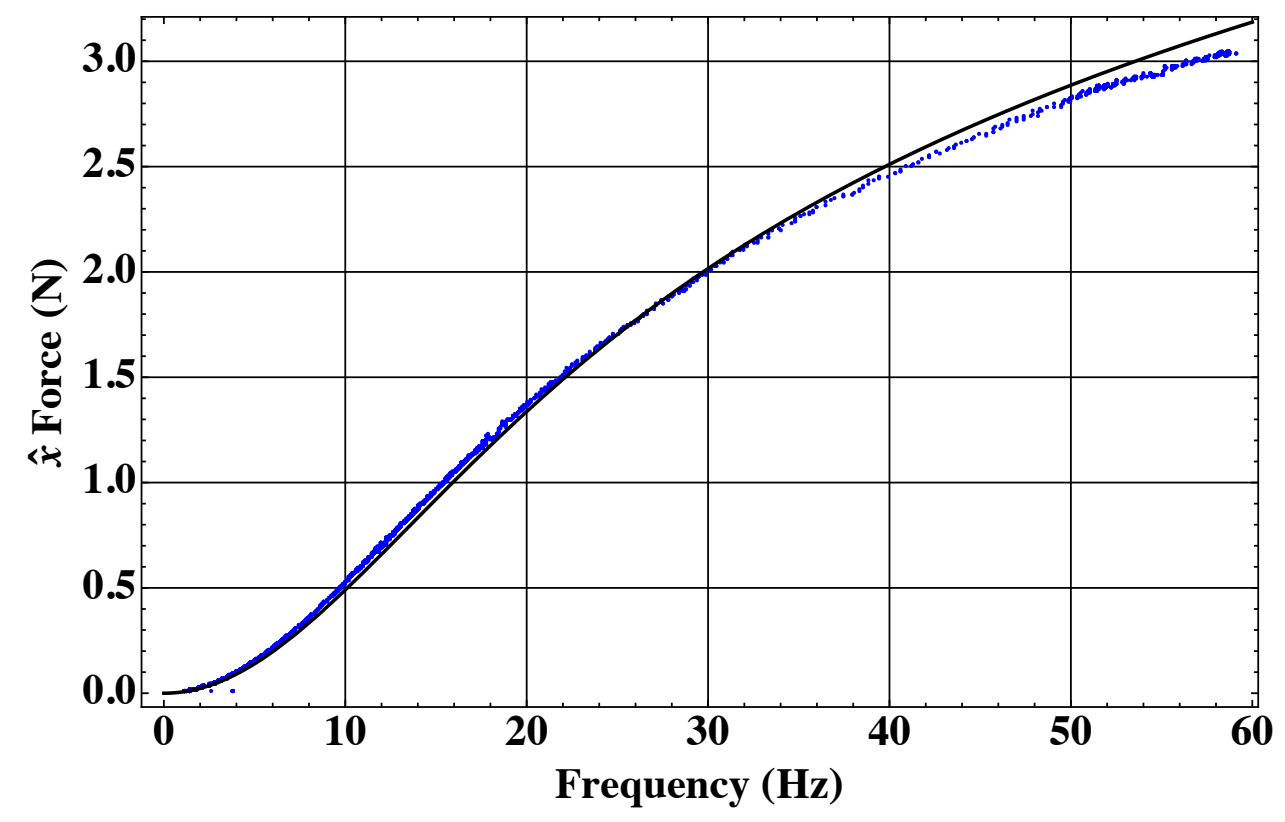

FIG. 6. Data (blue) and theory (black) showing the repulsive force.

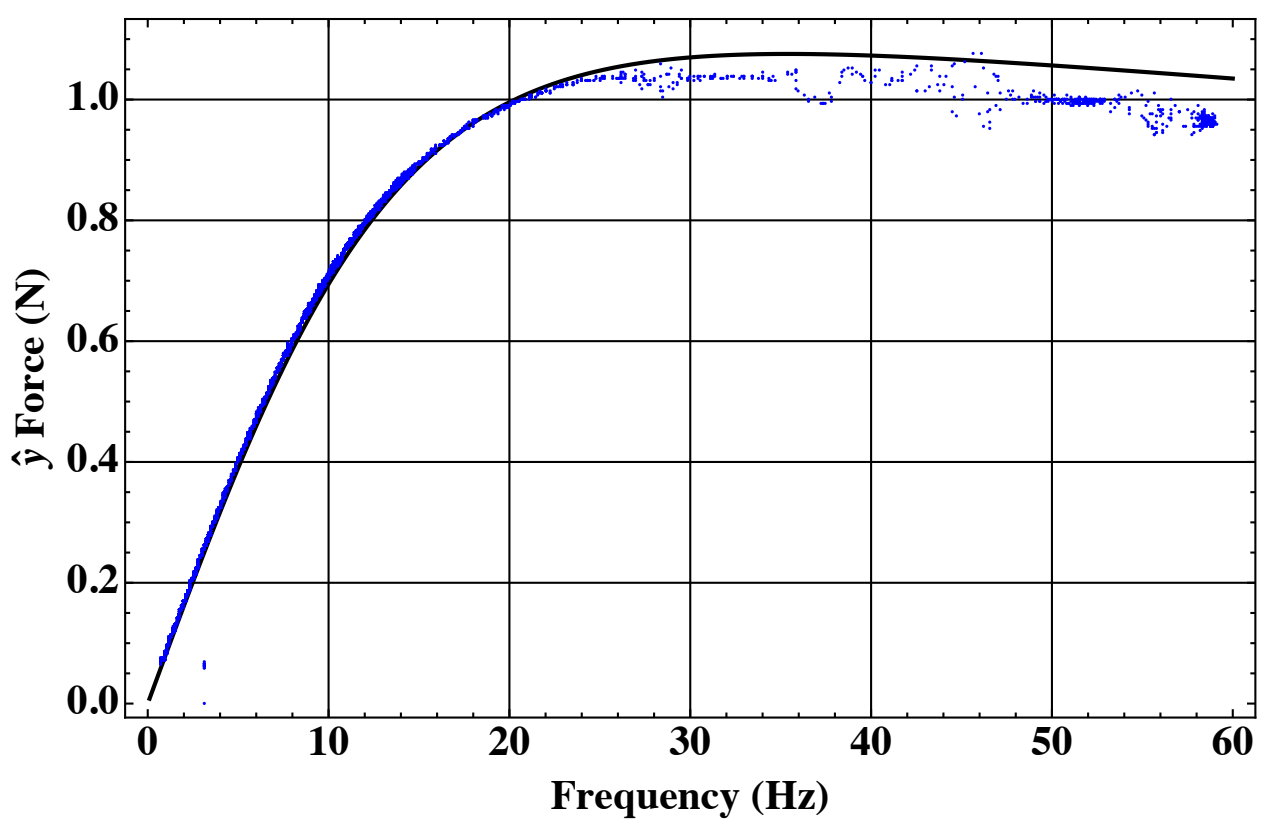

FIG. 7. Data (blue) and theory (black) showing the lateral force.

the dimensionless parameter, $r_{2} \sqrt{\mu_{0} \sigma \omega}$, to better illustrate the full range of force behavior. The first eddy current expression is responsible for generating the lateral drag force on the magnet while the sphere experiences an equal but opposite force in the $y$-direction. This 
force climbs and peaks and then drops off again with an increase in $r_{2} \sqrt{\mu_{0} \sigma \omega}$. Additionally, the non-uniformity in the force density across the sphere results in a decelerating torque. The second eddy current generates the repulsive force between the magnet and the sphere and continues to climb with $r_{2} \sqrt{\mu_{0} \sigma \omega}$, but levels off as the incoming magnetic field is becomes increasingly rejected inside the sphere by the described negative feedback process.

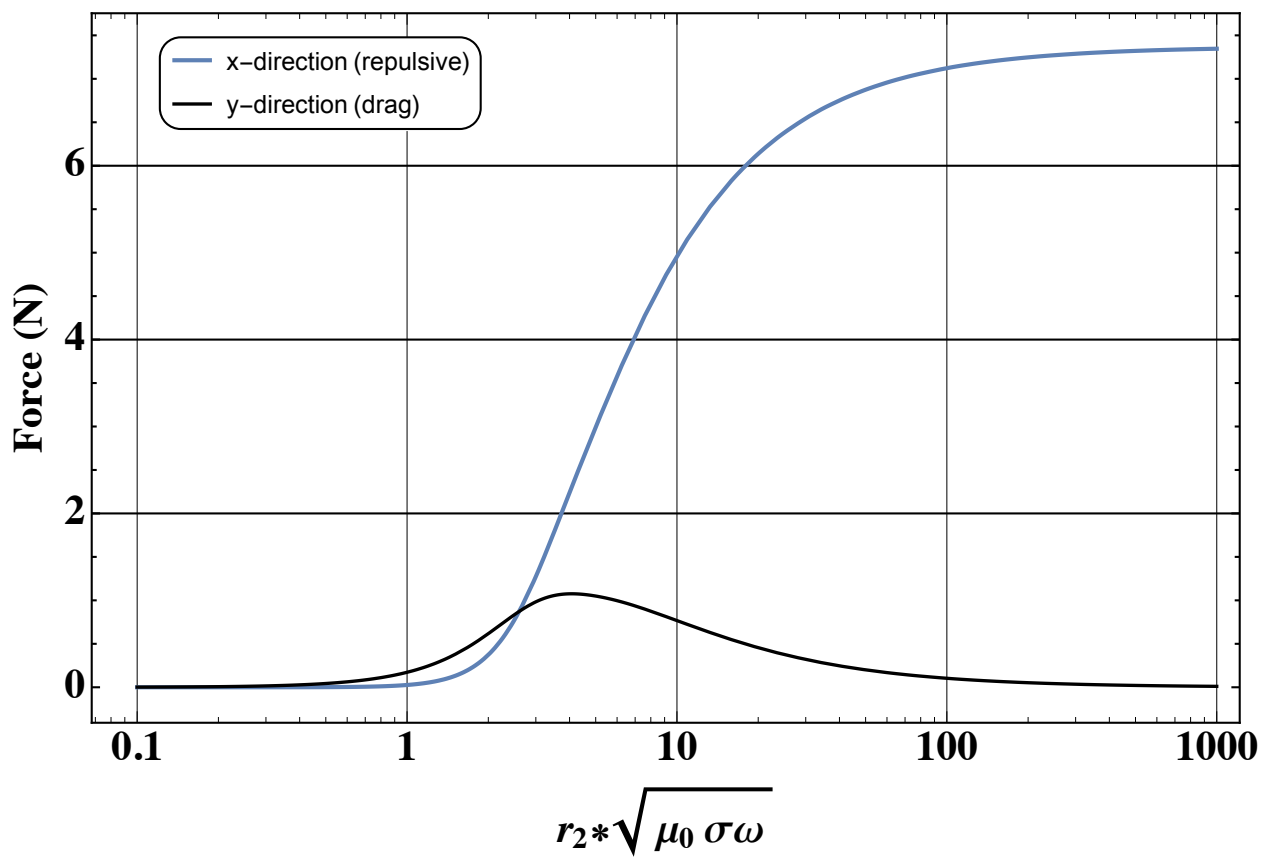

FIG. 8. These plots show the changes in the lateral and repulsive forces with an increase in $r_{2} \sqrt{\mu_{0} \sigma \omega}$. For these plots, $r_{2}, \mu_{0}$, and $\sigma$ were assumed to be constants associated with our test sphere.

\section{CONCLUSION}

It has been shown that the eddy currents generated by a fixed cylindrical magnet oriented along the equator of a spinning solid conducting sphere can be represented by two closed form expressions. These expressions where found by solving a fourth order differential equation with appropriate boundary conditions, yielding a solution in terms of Kelvin functions. The experiment showed close agreement between the measured forces and those computed with the derived expressions, confirming our revised version of the theory first worked out by Hertz. 


\section{Appendix A: THE MAGNETIC SCALAR POTENTIAL}

The magnetic scalar potential, $\Phi_{M}$, arises in regions of space where there are no time varying electric fields or source currents, allowing the magnetic field to be written as $\vec{B}=-\nabla \Phi_{M}$, where $\nabla^{2} \Phi_{M}=0$. As with the electric scalar potential, manipulating scalar functions is easier than vector valued functions, so one might suppose that the magnetic scalar potential would be commonly used. However, it is somewhat difficult to calculate for even a simple current loop and, as pointed out below, it is not a continuous function.

It can be shown that the magnetic scalar potential is given by the current in a closed loop times the solid angle of that loop as seen from a location in space. Analytically

$$
\Phi_{m}=\mu_{0} I \int_{A} \frac{\hat{n} \cdot \vec{d}}{d^{3}} d A
$$

where $I$ is the loop current, $A$ is the area of the loop, $\hat{n}$ is a unit normal out of the loop, and $\vec{d}$ is a vector connecting the observation point to a location on the surface of the loop, ${ }^{13}$ This interesting geometrical interpretation was known to Maxwell, who provided a form for the magnetic scalar potential for a round current loop, see Fig. 9, in terms of a series expansion $^{14}$ and after him other series expansions for the current loop were generated. ${ }^{15,16}$ At that time, it was preferable to represent functions as series expansions that could be calculated using look-up tables as opposed to using functions such as elliptical integrals. However, with modern computers closed form functional representations can be handled easily and are preferable. A more recent publication ${ }^{17}$ provides such a solution in cylindrical coordinates. It is presented below in spherical coordinates.

Define two parameters

$$
k=\frac{4 a r \sin \theta}{a^{2}+r^{2}+2 a r \sin \theta} \quad \text { and } \quad \beta=\frac{4 a r \sin \theta}{(a+r \sin \theta)^{2}},
$$

where $a$ is the radius of a current loop. The magnetic scalar potential for a round current loop, with current $I$, in spherical coordinates is

$$
\Phi_{m}=\left\{\begin{array}{lll}
\frac{\mu_{0} I}{2 \pi} \sqrt{1-k / \beta}(K(k)-\sqrt{1-\beta} \Pi(\beta, k)) & \text { when } \quad a<r \sin \theta \\
\frac{\mu_{0} I}{2 \pi} \sqrt{1-k / \beta}(K(k)+\sqrt{1-\beta} \Pi(\beta, k)) & \text { when } \quad a>r \sin \theta
\end{array}\right.
$$

where $K(k)$ and $\Pi(\beta, k)$ are complete elliptical integrals of the first and third kind, respectively. It should be pointed out that the scalar magnetic potential is not a continuous 


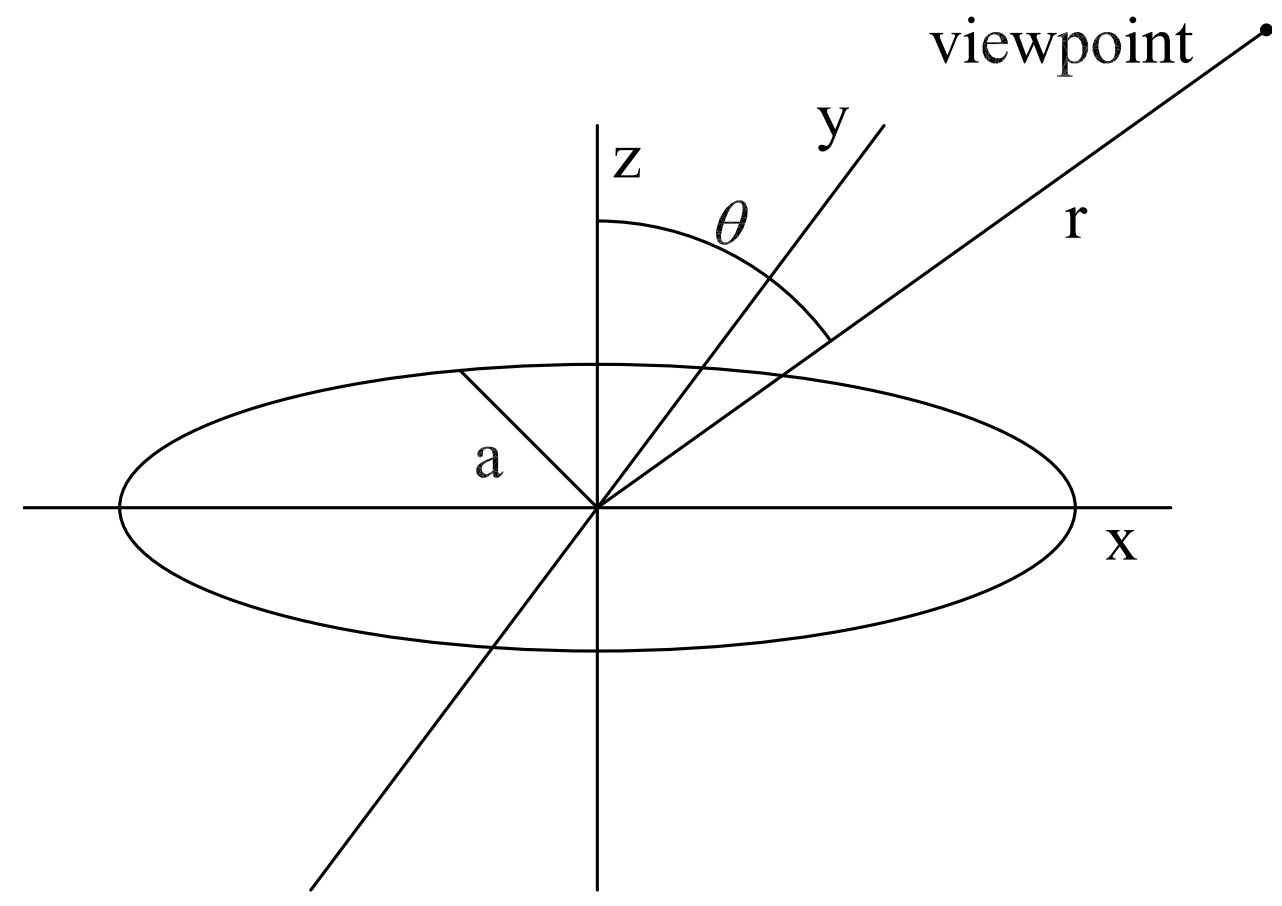

FIG. 9. A current loop showing a viewpoint location.

function. The magnetic field follows closed paths since the field lines must end on themselves, indicating that the magnetic scalar potential has positive (or negative) slope at all locations along one of these paths. However, a function cannot continuously increase (or decrease) along a closed path and end up with the same value. The resolution to this quandary is to first note that the magnetic scalar potential form shown in Eq. A3 is missing an arbitrary constant and that the sign (due to the square roots) is ambiguous. By adding constants and changing the sign of the function it can be made continuous, steadily increasing (or decreasing) until one passes through the area of the loop. Across this area, in the $x / y$ plane, the solid angle of the viewer changes from $2 \pi$ to $-2 \pi$ (or vice versa) reflecting the change in the sign of the dot product in Eq. A1. This discontinuity allows the magnetic field to continuously follow a closed path.

It can be verified with programs, such as Mathematica, that the gradient of Eqn. A-3, 
yields, to within a sign, the magnetic field for a current loop [18];

$$
\begin{gathered}
\vec{B}=\mu_{0} I \frac{a \cos \theta}{\pi\left(a^{2}+r^{2}-2 a r \sin \theta\right) \sqrt{a^{2}+r^{2}+2 a r \sin \theta}} E(k) \hat{r} \\
+\mu_{0} I \frac{\csc \theta}{2 \pi\left(a^{2}+r^{2}-2 a r \sin \theta\right) \sqrt{a^{2}+r^{2}+2 a r \sin \theta}}\left(\left(r^{2}+a^{2} \cos (2 \theta)\right) E(k)-\left(a^{2}+r^{2}-2 a r \sin \theta\right) K(k)\right) \hat{\theta}
\end{gathered}
$$

where $E(k)$ is the complete elliptical integral of the second kind. 
* Mark.A.Nurge@nasa.gov, (corresponding author)

$\dagger$ Robert.C.Yougquist@nasa.gov

1 Wikipedia, "Eddy current brake - Wikipedia, The Free Encyclopedia," 2017, https://en . wikipedia.org/w/index.php?title=Eddy_current_brake\&oldid=772624886.

2 Wikipedia, "Linear induction motor - Wikipedia, The Free Encyclopedia," 2017, https:// en.wikipedia.org/w/index.php?title=Linear_induction_motor\&oldid=775957988.

3 Wikipedia, "Electromagnetic Aircraft Launch System - Wikipedia, The Free Encyclopedia," 2017, https://en.wikipedia.org/w/index.php?title=Electromagnetic_Aircraft_ Launch_System\&oldid=769672993.

4 Wikipedia, "Maglev - Wikipedia, The Free Encyclopedia," 2017, https://en.wikipedia. org/w/index $\cdot$ php?title=Maglev\&oldid=776151802.

5 J. R. Reitz, "Forces on moving magnets due to eddy currents," Journal of Applied Physics, 41.5, 2067-2071, (1970).

6 J. R. Reitz and L. C. Davis, "Force on a rectangular coil moving above a conducting slab," Journal of Applied Physics, 43.4, 1547-1553, (1972).

7 A. Singh, "Theory of eddy-current brakes with thick rotating disc," Proceedings of the Institution of Electrical Engineers, IET Digital Library, 124, no. 4, (1977)

8 H. Hertz, Miscellaneous papers, (Macmillan and Co. Ltd, London, 1896), See Section 2, "On the induction in rotating spheres, 1880," pp. 35-126.

9 R. C. Youngquist et al., "A slowly rotating hollow sphere in a magnetic field: First steps to de-spin a space object.", American Journal of Physics, 84, no. 3, 181-191, (2016)

10 M. A. Nurge et al., "A thick-walled sphere rotating in a uniform magnetic field: The next step to de-spin a space object." American Journal of Physics, 85, no. 8, 596-610, (2017)

11 P. Moon and D. E. Spencer, Field Theory Handbook, 2nd Edition, (Springer-Verlag, Berlin, Heidelberg, 1988).

12 P. Lorrain and D. Corson, Electromagnetic Fields and Waves, 2nd Edition, (W. H. Freeman and Co, New York, NY 1970), See Section 9..8: "Magnetic Field Calculation."

13 M. Schwartz, Principles of electrodynamics, (Dover Publications, Inc., Mineola, NY, 1987), pp. 149-153. 
14 J. C. Maxwell, Electricity and Magnetism, (Clarendon Press, Oxford England. 1873), Vol. II, Secs. 694,695.

15 A. H. Jaffey, "Solid angle subtended by a circular aperture at point and spread sources: formulas and some tables," Review of Scientific Instruments, 25.4, 349-354, (1954).

16 M. W. Garrett, "Solid angle subtended by a circular aperture," Review of Scientific Instruments, 25.12, 1208-1211, (1954).

17 M. J. Prata, "Solid angle subtended by a cylindrical detector at a point source in terms of elliptic integrals," Radiation Physics and Chemistry, 67.5, 599-603, (2003).

18 J. C. Simpson et al., "Simple Analytic Expressions for the Magnetic Field of a Circular Current Loop," NASA Technical Memorandum, NASA/TM-2013-217919, (2013).

19 N. Praly et al., "Study on the eddy current damping of the spin dynamics of space debris from the Ariane launcher upper stages," Acta Astro., 76, 145-153, (2012).

20 F. Sugia et al., "Detumbling an uncontrolled satellite with contactless force by using an eddy current brake.", 2013 IEEE/RSJ International Conference on Intelligent Robots and Systems, 783-788, (2013)

21 N.O. Gómez and S.J. I. Walker, "Eddy currents applied to de-tumbling of space debris: Analysis and validation of approximate proposed methods," Acta Astro., 114, 34-53 (2015). 